

\title{
Nonlinear Coupling of Tearing Fluctuations in the Madison Symmetric Torus
}

\author{
J.S. Sarff, S. Assadi, a) A.F. Almagri, M. Cekic, D.J. Den Hartog, G. Fiksel, \\ S.A. Hokin, H. Ji, S.C. Prager, W. Shen, K.L. Sidikman, b) and M.R. Stoneking \\ University of Wisconsin, Madison, WI 53706
}

Three-wave, nonlinear, tearing mode coupling has been measured in the Madison Symmetric Torus (MST) reversed-field pinch (RFP) [Fusion Technol. 19, 131 (1991)] using bispectral analysis of edge magnetic fluctuations resolved in " $k$ space". The strength of nonlinear three-wave interactions satisfying the sum rules $m_{1}+m_{2}=m_{3}$ and $n_{1}+n_{2}=n_{3}$ is measured by the bicoherency. In the RFP, $m=1, n \sim 2 R / a$ ( 6 for MST) internally resonant modes are linearly unstable and grow to large amplitude. Large values of bicoherency occur for two $m=1$ modes coupled to an $m=2$ mode and the coupling of intermediate toroidal modes, e.g., $n=6$ and 7 coupled to $n=13$. These experimental bispectral features agree with predicted bispectral features derived from MHD computation. However, in the experiment, enhanced coupling occurs in the "crash" phase of a sawtooth oscillation concomitant with a broadened mode spectrum suggesting the onset of a nonlinear cascade.

a) Present address: Fermi National Accelerator Laboratory.

b) Present address: Oak Ridge National Laboratory. 


\section{INTRODUCTION}

Fluctuations in plasmas are observed at many frequencies and wavelengths. The processes which determine the evolution of turbulent spectra, such as the saturation of linear instability and the cascading of energy from unstable to damped waves, are inherently nonlinear. Large scale plasma behavior can also be influenced by nonlinear wave-wave interaction such as the generation of "coherent" structures, the turbulent generation of magnetic field (dynamo effect), and sawtooth oscillations. It is generally presumed the lowest order coupling of three waves is the dominant nonlinear wave-wave interaction. In this case, the three interacting waves satisfy the sum rule $\mathbf{k}_{1}+\mathbf{k}_{2}=\mathbf{k}_{3}$. The strength of such three-wave interaction can be estimated from experimental data using statistical bispectral analysis. This technique successfully identified three-wave interaction in fluids $1,2,3$ and nonlinear energy cascading in edge plasma fluctuations. ${ }^{4}$

Plasmas in the Madison Symmetric Torus ${ }^{5}$ (MST) are ideally suited for investigating nonlinear wave-wave processes since the largest amplitude fluctuations (tearing modes) strongly interact, and these fluctuations are well resolved using a large number of magnetic field sensors covering the plasma surface. These sensor arrays accurately resolve the spatial fluctuations in " $k$ space" and time. In this paper we present the results of bispectral analysis of tearing fluctuations applied directly in $k$-space. This analysis is applied to experimental data and, for comparison, to theoretical computational data derived from nonlinear, resistive, magneto-hydrodynamis (MHD) simulations. Experimental bispectra are presented for two different phases of the sawtooth oscillation-in betiveen and during the "crash". During the "crash" phase, enhanced cuupling is observed, suggesting the onset of a nonlinear cascade.

We are also interested in the radial transport caused by these global magnetic flucluations. In earlier work, the magnetic fluctuation induced 
nonambipolar particle transport given by the correlation of the total current density fluctuation and the radial magnetic field fluctuation was found to be small. 6 Here we will report recent measurements of the electron particle transport derived from the fluctuating electron current measured with an electrostatic energy analyzer. We also report preliminary results for the electron energy transport given by the fluctuating electron heat flux measured with a pyro-bolometric diagnostic.

MST is a large reversed-field pinch (RFP) with major radius, $R=1.5 \mathrm{~m}$, minor radius, $a=0.52 \mathrm{~m}$, toroidal plasma current, $I_{p} \leq 600 \mathrm{kA}$, and poloidal beta, $\beta_{\theta} \leq 20 \%$. The measurements reported in this paper were taken in lower current plasmas, $I_{p} \sim 250 \mathrm{kA}$ with reversal parameter, $F \approx-0.15$, pinch parameter, $\Theta \approx 1.7$, and edge safety factor, $q_{a} \approx-0.025$. Typically the data are selected at times near the peak in plasma current.

\section{OVERVIEW OF MAGNETIC FLUCTUATIONS}

Magnetic fluctuations are measured in MST with an extensive array of magnetic pickup coils. The bispectral analysis data were obtained from a 64station array of triplets $\left(B_{\phi}, B_{\theta}, B_{r}\right)$ uniformly distributed along the toroidal circumference (for the toroidal mode spectrum) and a 16-station array of triplets along the poloidal circumference (for the poloidal mode spectrum). The features of the magnetic fluctuations in MST have been reported in a number of papers, $6,7,8,9,10,22$ and only the results necessary for discussion of the bispectral measurements 11,12 are summarized here. The dominant magnetic fluctuations occur at frequencies $f \sim 20 \mathrm{kHz}$ with amplitudes $\widetilde{B} / B \sim 2 \%$ of the mean field. These dominant fluctuations are identified as $m=1, n \sim 2 R / a$ ( 6 in MST) tearing modes. The spectral characteristics of the experimental magnetic fluctuations quantitatively agree with the characteristics of theoretical magnetic fluctuations 
simulated by a $3 D$, resistive MHD code, ${ }^{13}$ except the experimental amplitudes are smaller than the theoretical amplitudes. In part, this results from a difference in the Lundquist numbers of the computation $\left(S \sim 10^{4}\right)$ and experiment $\left(S \sim 10^{6}\right)$. An example of the Fourier series decomposition of the fluctuations measured by the 64-coil toroidal array is shown in Fig. 1. (Note that modes 15-32 are not shown.) Only the amplitudes of the modes are shown in this figure. Not shown are the phases of the modes which change at the characteristic frequencies $\sim 20 \mathrm{kHz}$ as the plasma rotates in the laboratory frame of reference. The slow variation ( $2 \mathrm{~ms}$ ) of the amplitudes corresponds to changes during the phase of a sawtooth oscillation. ${ }^{14,15}$ In this example, a sawtooth "crash" occurs at $20.5 \mathrm{~ms}$, easily identified by the sudden increase in the low and high $n$-mode amplitudes. The bispectra discussed in the following section were obtained for a time between sawtooth "crash" events when the mode spectrum is relatively narrow and for a time near the sawtooth "crash" when the spectrum is broadened.

The tearing mode fluctuations are phase-locked to each other.8,9,10 This is illustrated in Fig. 2(a) by the tendency for a spatially localized magnetic structure to appear in the "raw" data and more explicitly in Fig. 2(b) where the normalized modes, $\cos \left[n \phi+\delta_{n l}(t)\right]$, for $n=6-10$ are plotted. In Fig. 2(a), the box cursor identifies the location of the maximum fluctuation. This "bump" rotates toroidally in the ion diamagnetic drift direction at a speed $\sim 3 \times 10^{6} \mathrm{~cm} / \mathrm{s}$. The normalized modes in Fig. 2(b) are plotted at $50 \mu$ s intervals and clearly illustrate the phase alignment of the composite modes at exactly the location of the localized structure. As discussed in the next section, such phase-locking of waves characterizes nonlinear coupling, so the phase-locking of MST tearing modes is the first evidence for the existence of their nonlinear coupling. Phase-locking of tearing fluctuations has been observed in several other RFP devices and is apparently a ubiquitous feature of RFP plasmas. ${ }^{16,17,18}$ Also, the spatially localized structure 
formed by this nonlinear interaction exemplifies a "coherent structure" alluded to in the introduction.

\section{NONLINEAR COUPLING OF TEARING MODES}

We measure the nonlinear coupling of tearing modes in MST using bispectral analysis directly in $k$-space. This is an extension of previous bispectral analysis in frequency space. ${ }^{4,11}$ Before discussing these results, the definition for and interpretation of bicoherency will be presented. ${ }^{19}$

\section{A. Definition and interpretation of bicoherency}

Quadratic nonlinear coupling of three Fourier modes is measurable by the bicoherence, $b\left(n_{1}, n_{2}, n_{3}\right)$, defined as

$$
b\left(n_{1}, n_{2}, n_{3}\right)=\frac{\left\langle B^{*}\left(n_{1}\right) B^{*}\left(n_{2}\right) B\left(n_{3}\right)\right\rangle}{\sqrt{\left\langle B^{2}\left(n_{1}\right) B^{2}\left(n_{2}\right) \backslash B^{2}\left(n_{3}\right)\right\rangle}}
$$

where, in this case, the toroidal modes satisfy the sum rule $n_{3}=n_{1}+n_{2}$. Similar analysis applies to the poloidal modes. In this definition, $B(n)$ represents the magnitude and phase of the $n^{\text {th }}$ mode in a Fourier series representation of the magnetic fluctuation measured by the pickup coil arrays at the surface of the plasma, and \langle\rangle denotes an ensemble average. The bicoherence is normalized such that $\left|b\left(n_{1}, n_{2}, n_{3}\right)\right| \leq 1$. If the three modes $n_{1}, n_{2}$ and $n_{3}$ are not nonlinearly coupled, $\left|b\left(n_{1}, n_{2}, n_{3}\right)\right|=0$. If all of the fluctuation in mode $n_{3}$ is nonlinearly coupled to modes $n_{1}$ and $n_{2}$, then $\left|b\left(n_{1}, n_{2}, n_{3}\right)\right|=1$. To see that non zero bicoherence results from phase-locking of the three modes, write $B(n)=\left|B_{n}\right| e^{-i \delta_{n}}$ where $\delta_{n}$ is the spatial phase. Then

$$
\left\langle B^{*}\left(n_{1}\right) B^{*}\left(n_{2}\right) B\left(n_{3}\right)\right\rangle=\left\langle\left|B_{n 1}\right|\left|B_{n 2}\right|\left|B_{n 3}\right| e^{i\left(\delta_{n 1}+\delta_{n 2}-\delta_{n 3}\right)}\right\rangle .
$$


If the phase factor $\delta_{n 1}+\delta_{n 2}-\delta_{n 3}$ does not have a statistically stationary relationship, the bicoherence is zero. Phase-locking means a fixed relationship exists between the phases of the modes. Such phase-locking can result from quadratic nonlinear coupling, i.e.,

$$
B\left(n_{3}\right) \propto B\left(n_{1}\right) B\left(n_{2}\right)=\left|B_{n 1}\right|\left|B_{n 2}\right| e^{-i\left(\delta_{n 1}+\delta_{n 2}\right)}
$$

and $\delta_{113}=\delta_{n 1}+\delta_{n 2}$. This example illustrates nonlinear generation of mode $n_{3}$ by quadratic coupling of modes $n_{1}$ and $n_{2}$ in a "sum" interaction, i.e., $n_{3}=n_{1}+n_{2}$. In the "difference" interaction, $n_{3}=n_{1}-n_{2}, B\left(n_{3}\right) \propto B\left(n_{1}\right) B^{*}\left(n_{2}\right)$ and $\delta_{n 3}=\delta_{n 1}-\delta_{112}$.

Even though three given modes may be entirely nonlinearly coupled, the bicoherence for this interaction can have a value less than unity if the mode $n_{3}$ is nonlinearly coupled in more than one three-wave interaction. The normalized power in the mode $n_{3}$ can be written

$$
\frac{B\left(n_{3}\right)}{\sqrt{\left\langle B^{2}\left(n_{3}\right)\right\rangle}}=\frac{B^{u n c}\left(n_{3}\right)}{\sqrt{\left\langle B^{2}\left(n_{3}\right)\right\rangle}}+\sum_{\substack{n_{1}^{\prime}, n_{2}^{\prime} \\ n_{1}^{\prime}+n_{2}^{\prime}=n_{3}}} b\left(n_{1}^{\prime} n_{2} n_{2}^{\prime}, n_{3}\right) \frac{B\left(n_{1}^{\prime}\right) B\left(n_{2}^{\prime}\right)}{\sqrt{\left\langle B^{2}\left(n_{1}^{\prime}\right) B^{2}\left(n_{2}^{\prime}\right)\right\rangle}}
$$

where $B^{u n c}\left(n_{3}\right)$ is the fluctuation uncolipled to other modes, and the summation term is the fluctuation quadratically coupled to other modes. In this summation, $b\left(n_{1}, n_{2}, n_{3}\right)$ represents the quadratic coupling coefficient and is identical to the bicoherence when four-wave and higher order nonlinear coupling processes are reducible to three-wave coupling processes. To see this, multiply by $B^{*}\left(n_{1}\right) B^{*}\left(n_{2}\right)$ and ensemble average. The assumption $\left\langle B\left(n_{1}^{\prime}\right) B\left(n_{2}^{\prime}\right) B^{*}\left(n_{1}\right) B^{*}\left(n_{2}\right)\right)$ is non zero only when $n_{1}^{\prime}=n_{1}$ and $n_{2}^{\prime}=n_{2}$ ignores higher order coupling.

If instead we multiply by $B^{*}\left(n_{3}\right)$ and ensemble average, a conservation-like equation for the normalized fluctuation power in mode $n_{3}$ is derived: 


$$
1=\frac{\left\langle B^{*}\left(n_{3}\right) B^{u n c}\left(n_{3}\right)\right\rangle}{\left\langle B^{2}\left(n_{3}\right)\right\rangle}+\sum_{\substack{n_{1}^{\prime}, n_{2}^{\prime} \\ n_{1}^{\prime}+n_{2}^{\prime}=n_{3}}} b^{2}\left(n_{1}^{\prime}, n_{2}^{\prime}, n_{3}\right)
$$

Even in the case when all of the power in mode $n_{3}$ is nonlinearly coupled $\left(B^{u n c}\left(n_{3}\right)=0\right)$, if the summation has more than one term which occurs when the mode $n_{3}$ is involved in more than one three-wave interaction, then the squared bicoherency of each term is less than unity. The sum of all squared bicoherencies involving mode $n_{3}$ measures the total nonlinear behavior of that mode. This fact will be important when comparing the measured bispectra with MHD modeling of the RFP.

\section{B. Comparison of experimental and theoretical bispectra}

In the following, the experimental bispectra represent the ensemble average of 256 data records, each $256 \mu \mathrm{sec}$ in duration, selected from 40 identical discharges. For the toroidal spectra, 64 magnetic pickup coils resolve $n<32$, and for the poloidal spectra, 16 coils resolve $m<8$. Note that the coil array is not two dimensional, i.e., each $n$-mode includes contributions from all $m$-modes and vice versa. The theoretical bispectra are calculated from MHD simulation data evaluated at $r=a$, as in the experiment. The initial value code ${ }^{13}$ solves the compressible, resistive MHD equations in a periodic cylinder with aspect ratio 3 , as in the experiment. The code includes $|m|<2$ and $|n|<42$. To save computational time, the Lundquist number for the theoretical data is $S=10^{4}$ whereas in the experiment $S \sim 10^{6}$. An ensemble average of 256 code runs was assembled, each run initialized by randomizing the phases of the magnetic and velocity fluctuations. The data shown below have been previously reported ${ }^{12}$ and are summarized here for discussion. 
The squared bicoherencies of poloidal mode couplings are shown in Fig. 3. These data represent the time between sawtooth "crash" events. The squared bicoherency of the interaction $m_{1}+m_{2}=m_{3}$ is recorded vertically from the $\left(m_{1}, m_{2}\right)$ plane. The symmetries associated with exchange of indices and parity allow the rules $m_{2} \geq\left|m_{1}\right| \geq 0$ when plotting the squared bicoherency, and Nyquist limits must also be observed. In both the experiment (Fig. 3a) and the MHD theory (Fig. 3b), large bicoherency is observed for two $m=1$ modes coupled to an $m=2$ mode, i.e., $m_{1}=1, m_{2}=1, m_{3}=2$ and $m_{1}=-1, m_{2}=2, m_{3}=1$. The sign of $m$ (or $n$ ) indicates direction of propagation.

The squared bicoherencies of toroidal mode couplings are shown in Fig. 4. Unlike the poloidal mode coupling, toroidal mode coupling occurs between many modes, reflecting the breadth of the n-spectrum. A difference between the experimental (Fig. 4a) and theoretical (Fig. 4b) coupling is the relative breadth of the experimental coupling. This may, in part, be due to the different Lundquist numbers. In both cases, however, the squared bicoherencies of "sum" interactions of intermediate modes (e.g., $\left.n_{1}=6, n_{2}=7, n_{3}=13\right)$ are largest. The "difference" interactions of these modes, found along the $n_{1}=1,2, \ldots$ lines, appear to be weaker.

Over the years, a detailed theoretical picture of the nonlinear coupling of tearing modes has emerged from careful diagnosis of the MHD simulations of the RFP.20 The coupling of magnetic and velocity fluctuations is the heart of the MHD dynamo. The primary tearing instability results from resistive diffusion of the equilibrium and generates the core resonant modes $m=1, n \sim 2 R / n$ ( 6 in MST). These modes nonlinearly couple in both "sum" and "difference" interactions. The "sum" interaction generates $m=2, n \gtrsim 4 R / a$ modes which cascade to short scale and are dissipated. The "difference" interaction generates $m=0, n \sim 1$ modes. The effect of the $m=0$ modes is complicated. First, they all resonate at the toroidal 
field reversal surface $(q=0)$ near the edge of the plasma. The large island that forms quasi-linearly flattens the equilibrium profile and destabilizes $m=1$, large- $n$ modes near the reversal surface. Second, the $m=0$ modes couple to these new $m=1$ modes generating more $m=1$ modes which are stable and dissipative.

In this MHD picture, the $m=0$ modes degenerately couple to many $m=1$ modes. Since the quasi-steady-state equilibria tend to be $m=0,2$ stable, the $m=0,2$ fluctuations are mostly nonlinear. To the extent the experiment obeys MHD, the squared bicoherencies involving $n \sim 1$ modes should be small since the sum of all three-mode interactions involving the low $-n$ modes is order unity. In contrast, the $m=2$ coupling branch generates unique $n$-modes; the squared bicoherencies for the "sum" interaction of the dominant unstable modes $n \sim 2 R / a$ should be large. Qualitatively this characterizes the toroidal mode coupling bicoherencies shown in Fig. 4. Unfortunately, the lack of single helicity identification of the experimental fluctuations prevents an unambiguous quantitative comparison of the experiment and MHD theory. We note, however, that the observed phaselocking of the $m=1, n \sim 6$ modes in the experiment (Fig. 2) is more likely mediated by $m=0$ coupling than $m=2$ coupling since all of the $m=1, n \sim 6$ modes can be coupled to the same $m=0, n \sim 1$ modes, i.e., the modes $m=1, n$ and $m=1, n+1$ couple to $m=0, n=1$, the modes $m=1, n$ and $m=1, n+2$ couple to $m=0, n=2$, etc... In this case, the phase of the magnetic "bump" is just the phase of the $m=0$ modes.

The data above are for times between sawtooth "crash" events. During the "crash", enhanced coupling is observed. The squared bicoherencies for the "crash" phase are shown in Fig. 5. In addition to the coupling of two $m=1$ and an $m=2$ mode, strong coupling of two $m=2$ and an $m=4$ mode is observed. There are also subdominant peaks for the coupling of the $m=1,3$ and 4 modes. Strong coupling of toroidal modes occurs between all resolved modes, consistent with the broadened spectrum observed during the "crash" as in Fig. 1. The enhanced 
nonlinear coupling during this phase of the sawtooth oscillation strongly suggests an onset of nonlinear cascading rather than suciden destabilization of many modes resulting from sudden changes in the equilibrium profiles.

\section{MAGNETIC FLUCTUATION INDUCED TRANSPORT}

The correlations of fluctuating quantities which can yield radial transport are listed in Table 1. These correlations are classified as electrostatic, where radial transport is convected by the fluctuating radial $\widetilde{\mathbf{E}} \times \mathbf{B}$ particle drift, or as magnetic, where radial transport results from parallel streaming along stochastic field lines. The electrostatic terms have been measured in the edge of MST. ${ }^{21,22}$ The electrostatic-fluctuation-induced particle transport accounts for a large fraction of the global particle transport, but the electrostatic-fluctuation-induced energy transport is small compared to global energy transport.

The magnetic-fluctuation-induced nonambipolar particle transport has been measured to be small in the edge. ${ }^{6}$ Here we report recent results for electron transport measured by correlating the fluctuating electron current and magnetic field. The electron current is measured in the edge of MST with a two-channel, pinhole, electrostatic energy analyzer. ${ }^{23}$ This diagnostic measures the current on a magnetic field line, both in the "forward" and the "backward" directions. Pickup coils measure the fluctuating magnetic field at the location of the current measurement. The frequency resolved cross-coherence and cross-phase are shown in Fig. 6. The current measurement excludes low energy electrons $(\$ 50 \mathrm{eV})$ due to hardware limitations; in the future, we hope to include all electron energies in the measurement. For frequencies $\sim 20 \mathrm{kHz}$, corresponding to the tearing mode fluctuations, the cross-phase is $\pi / 2$ giving no transport. Integrating over the frequency band $15-25 \mathrm{kHz}$ where there is statistically significant coherence, the electron transport is less than $5 \%$ of the global particle 
transport. Recalling the previous result of negligible nonambipolar particle transport, we conclude the magnetic-fluctuation-induced ion transport in the edge is small as well, assuming the electron current is dominated by electrons with energy $\gtrsim 50 \mathrm{eV}$.

We are also beginning to measure the fluctuating electron parallel heat flux which, correlated with the magnetic field fluctuation, directly yields the magnetic-fluctuation-induced electron heat transport. The measurement of the heat flux is given by a pyro-electric bolometer diagnostic. ${ }^{24}$ Like the electron energy analyzer, is has two channels for measuring the heat flux from both the "forward" and "backward" directions on a magnetic field line. The pyro-electric bolometer uses gold plated $\mathrm{LiTiO}_{3}$ crystals as sensors and has a bandwidth of $\sim 50 \mathrm{kHz}$. Frequency power spectra for the "forward" and "backward" heat fluxes are shown in Fig. 7; the fluctuations peak near the tearing mode frequencies. The first estimate using the measured heat flux for the magneticfluctuation-induced electron heat transport accounts for $\sim 30 \%$ of the nonradiated global heat flux at the edge. Work is in progress to quantify the transparency of the pinhole estimated to be $\sim 50 \%$, so the preliminary best estimate for the magnetic-fluctuation-induced electron energy flux is $\sim 60 \%$ of the non-radiated global heat flux.

\section{SUMMARY}

Quadratic nonlinear coupling of tearing modes has been measured in MST using bispectral analysis. Comparison of the coupling of RFP tearing modes in the experiment to detailed theoretical predictions developed in recent years is facilitated by directly comparing experimental and theoretical bicoherency. The experimental and theoretical bicoherency qualitatively agree, but the coupling of toroidal modes is generally more broad in the experiment. In the "crash" phase 
of a sawtooth oscillation, the coupling of both poloidal and toroidal modes broadens. The couplings of unstable $m=1, n \sim 6$ core resonant modes to $m=2, n \sim 15$ modes have the largest values of bicoherency. The couplings of these same unstable modes to $m=0, n \sim 1$ modes have relatively small bicoherency, but this is consistent with the expectation of degeneracy for the $m=0$ couplings. We note that the observed phase-locking of the dominant unstable modes $m=1, n \sim 6$ is more likely mediated by the $m=0$ cuupling branch because of this degeneracy.

Measurement of fluctuation-induced transport is ongoing in MST. Preliminary measurements indicate that the magnetic-fluctuation-induced electron transport is small in the edge. This result and the previous result for small nonambipolar magnetic-fluctuation-induced transport imply the ion magnetic-fluctuation-induced transport is small in the edge as well. The fluctuating parallel heat flux is measured in MST using a pyro-bolometric diagnostic. Preliminary measurements of the electron magnetic-fluctuationinduced energy flux indicate it is a substantial fraction of the non-radiated energy flux at the edge.

\section{ACKNOWLEDGMENTS}

The authors are grateful to Dr. Gary Craddock, Dr. Y.L. (Bill) Ho, Dr. Dalton Schnack, Carl Sovinec, and Dr. Paul Terry for useful discussions. S.A. is indebted to Dr. John Marriner and Dr. Patrick Colestock for valuable discussions.

This work was supported by the US Department of Energy. Additional support was provided by the AT\&T Corporation and the National Science Foundation for Nonlinear Studies. 


\section{REFERENCES}

${ }^{1}$ K. Hasselmann, W. Munk, and G. MacDonald, in Proceedings of the Symposium of Time Series Analysis, edited by M. Rosenblatt (Wiley, New York, 1963), p. 125.

2 R.W. Miksad, F.L. Jones, and E.J. Powers, Phys. Fluids 23, 258 (1983).

3 S. Elgar and R.T. Guza, J. Fluid Mech. 161, 425 (1985).

4 Ch.P. Ritz, E.J. Powers, and R.D. Bengston, Phys. Fluids B 1, 153 (1989).

${ }^{5}$ R.N. Dexter, D. Kerst, T.W. Lovell, S.C. Prager, and J.C. Sprott, Fusion Tech. 19, 131 (1991).

6W. Shen, R.N. Dexter, and S.C. Prager, Phys. Rev. Lett. 68, 1319 (1992).

${ }^{7}$ S. Assadi, J.A. Beckstead, R.N. Dexter, S.C. Prager, J.S. Sarff, W. Shen, and J.C. Sprott, Bull. Amer. Phys. Soc. 34, 2107 (1989).

${ }^{8}$ A. Almagri, S. Assadi, J. Beckstead, G. Chartas, N. Crocker, D. DenHartog, R. Dexter, S. Hokin, D. Holly, E. Nilles, S. Prager, T. Rempel, J. Sarff, E. Scime, C. Spragins, J. Sprott, G. Starr, M. Stoneking, and C. Watts, in Proceedings of the Workshop on Physics of Alternative Confinement Schemes, Varenna Italy, 1990, edited by S. Ortolani and E. Sidoni (Societá Italian di Fisica, Bologna, 1991) p. 223.

${ }^{9}$ J.S. Sarff, A.F. Almagri, S. Assadi, J.A. Beckstead, R.N. Dexter, and S.C. Prager, Bull. Amer. Phys. Soc. 35, 2010 (1990).

${ }^{10}$ A.F. Almagri, S. Assadi, S.C. Prager, J.S. Sarff, D.W. Kerst, Locked Modes and Magnetic Field Errors in MST, to be published in Phys. Fluids.

11S. Assadi, A.F. Almagri, J.A. Beckstead, S.A. Hokin, S.C. Prager, J.S. Sarff, E. Scime, and K.L. Sidikman, Bull. Amer. Phys. Soc. 35, 2010 (1990). 12S. Assadi, S.C. Prager, and K.L. Sidikman, Phys. Rev. Lett. 69, 281 (1992). ${ }^{13}$ D.D. Schnack, D.C. Barnes, Z. Mikic, D.S. Harned, and E.J. Caramana, J. Comput. Phys. 70, 330 (1987). 
14J.A. Beckstead, Sawteeth in the MST Reversed Field Pinch, Ph. D. thesis, University of Wisconsin-Madison, 1990.

15S. Hokin, A. Almagri, S. Assadi, J. Beckstead, G. Chartas, N. Crocker, M.

Cudzinovic, D. Den Hartog, R. Dexter, D. Holly, S. Prager, T. Rempel, J. Sarff,

E. Scime, W. Shen, C. Spragins, G. Starr, M. Stoneking, and C. Watts, Phys.

Fluids B 3, 2241 (1991).

16T. Tamano, R.J. LaHaye, T.N. Carlstrom, P.L. Taylor, and M.J. Schaffer, Phys. Rev. Lett. 59, 1444 (1987).

17K. Hattori, Y. Hirano, T. Shimada, Y. Yagi, Y. Maejima, I. Hirota, and K. Ogawa, Phys. Fluids B 3, 3111 (1991).

18P. Greene and S. Robertson, submitted to Phys. Fluids.

${ }^{19}$ For a more detailed discussion of the bispectrum and methods for its estimation, see, for example, D.R. Brillinger and M. Rosenblatt, in Spectral Analysis of Time Series, edited by B. Harris (Wiley, New York, 1967) and M.B. Priestly, Spectral Analysis and Time Series (Academic, London, 1981). 20Y.L. Ho and G. Craddock, Phys. Fluids B 3, 721 (1991) and references therein. ${ }^{21}$ T.D. Rempel, C.W. Spragins, S.C. Prager, S. Assadi, D.J. Den Hartog, and S. Hokin, Phys. Rev. Lett. 67, 1438 (1991).

22T.D. Rempel, A.F. Almagri, S. Assadi, D.J. Den Hartog, S.A. Hokin, S.C. Prager, J.S. Sarff, W. Shen, K.L. Sidikman, C.W. Spragins, J.C. Sprott, M.R. Stoneking, and E.J. Zita, Phys. Fluids B 4, 2136 (1992).

${ }^{23}$ M. Stoneking, S. Hokin, W. Shen, and G. Fiksel, Bull. Amer. Phys. Soc. 37, 1606 (1992).

${ }^{24}$ G. Fiksel, S.C. Prager, and W. Shen, Bull. Amer. Phys. Soc. 37, 1606 (1992). 
Electrostatic

Particle
Transport

Current

Transport

Energy

Transport

$$
\frac{\left\langle\tilde{n}_{i, e} \widetilde{E}_{\perp}\right\rangle}{B_{o}}
$$

$\frac{\left\langle\tilde{J}_{\|} \widetilde{E}_{\perp}\right\rangle}{B_{o}}$

$\frac{\left\langle\widetilde{p}_{e} \widetilde{E}_{\perp}\right\rangle}{B_{0}}$
Magnetic

$$
\frac{\left\langle\tilde{J}_{\| i, e} \widetilde{B}_{r}\right\rangle}{e B_{o}}
$$

$$
\frac{e}{m_{e}} \frac{\left\langle\tilde{p}_{\| l} \tilde{B}_{r}\right\rangle}{B_{o}}
$$

$$
\frac{\left\langle\widetilde{q}_{\| e} \widetilde{B}_{r}\right\rangle}{B_{o}}
$$

Table 1. Fluctuation-induced-transport fluxes resulting from quadratic correlations. Negligible mean electron drift is assumed. 


\section{FIGURE CAPTIONS}

1. Amplitudes of the $n \leq 14$ toroidal modes in a Fourier series representation of the poioidal magnetic field fluctuation $\left(\widetilde{B}_{\theta}\right)$ from the toroidal array of 64 pickup coils. A sawtooth oscillation "crash" event occurs at $20.5 \mathrm{~ms}$. $\widetilde{B}_{\theta}^{r m s}$ is the root-mean-square spatial average fluctuation amplitude.

2. Phase-locking of the dominant $m=1, n \sim 2 R / a$ Fourier modes detected (a) by spatially localized rotating "bump" in $\widetilde{B}_{\theta}$ data and (b) explicitly by the Fourier phase alignment of the dominant modes at the location of the "bump". These data are the same as in Fig. 1.

3. Squared bicoherency of the coupling of poloidal modes, $m_{1}+m_{2}=m_{3}$, (a) in MST data and (b) in MHD theoretical data. The value of the squared bicoherency for the coupling $m_{1}+m_{2}=m_{3}$ is recorded vertically from the $\left(m_{1}, m_{2}\right)$ plane. These data are for the poloidal component $\left(\widetilde{B}_{\theta}\right)$ of the magnetic fluctuation. The maximum value of the squared bicoherency is 0.35 in (a) and 0.60 in (b).

4. Squared bicoherency of the coupling of toroidal modes, $n_{1}+n_{2}=n_{3}$, (a) in MST data and (b) in MHD theoretical data. The plotting scheme is as for Fig. 3, except these data are for the toroidal component $\left(\widetilde{B}_{\phi}\right)$ of the magnetic fluctuation. The maximum value of the squared bicoherency is $0.45 \mathrm{in}$ (a) and $0.61 \mathrm{in}(\mathrm{b})$.

5. Squared bicoherency of the coupling of (a) poloidal modes and (b) toroidal modes during a sawtooth oscillation "crash" event in MST. The maximum value of the squared bicoherency is 0.52 in (a) and 0.42 in (b). 
6. Cross-spectral properties of parallel electron current fluctuations $\left(\widetilde{J}_{\| e}\right)$ measured by an electrostatic electron energy analyzer and radial magnetic field fluctuations $\left(\widetilde{B}_{r}\right)$ detected with a magnetic pickup coil. The crosscoherence is shown in (a) and the cross-phase in (b). The current includes electrons with energy $\gtrsim 50 \mathrm{eV}$ and was collected at radius $r / a=0.95$.

7. Frequency power spectra for the "forward" $\left(\tilde{q}_{\| f}\right)$ and "backward" $\left(\tilde{q}_{\| b}\right)$ fluctuating parailel electron heat flux measured with a pyro-bolometer. The fluctuation is peaked at low frequency, near the dominant tearing modes. 


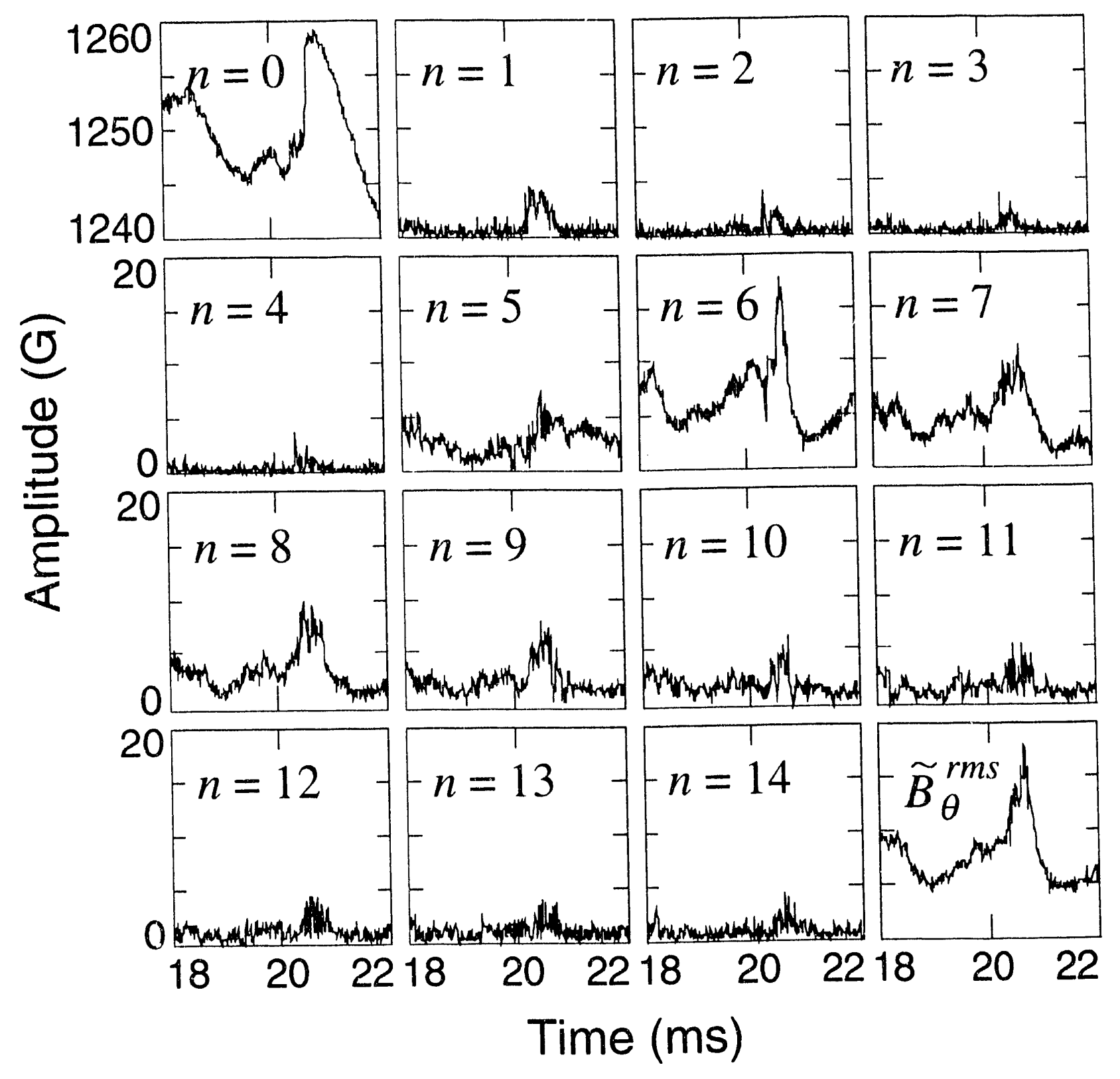

Fig. 1 


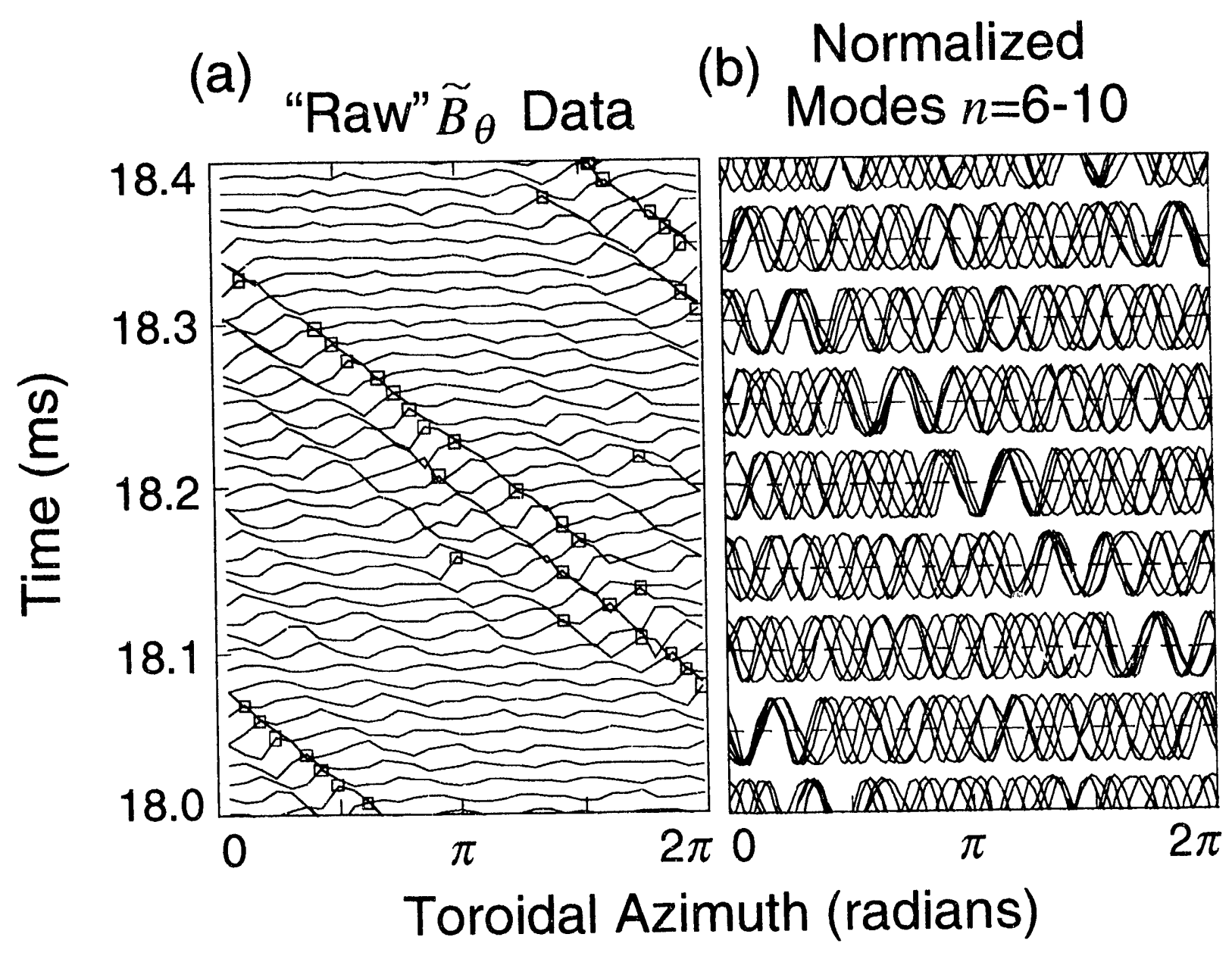

Fig. 2 
(a) Experiment
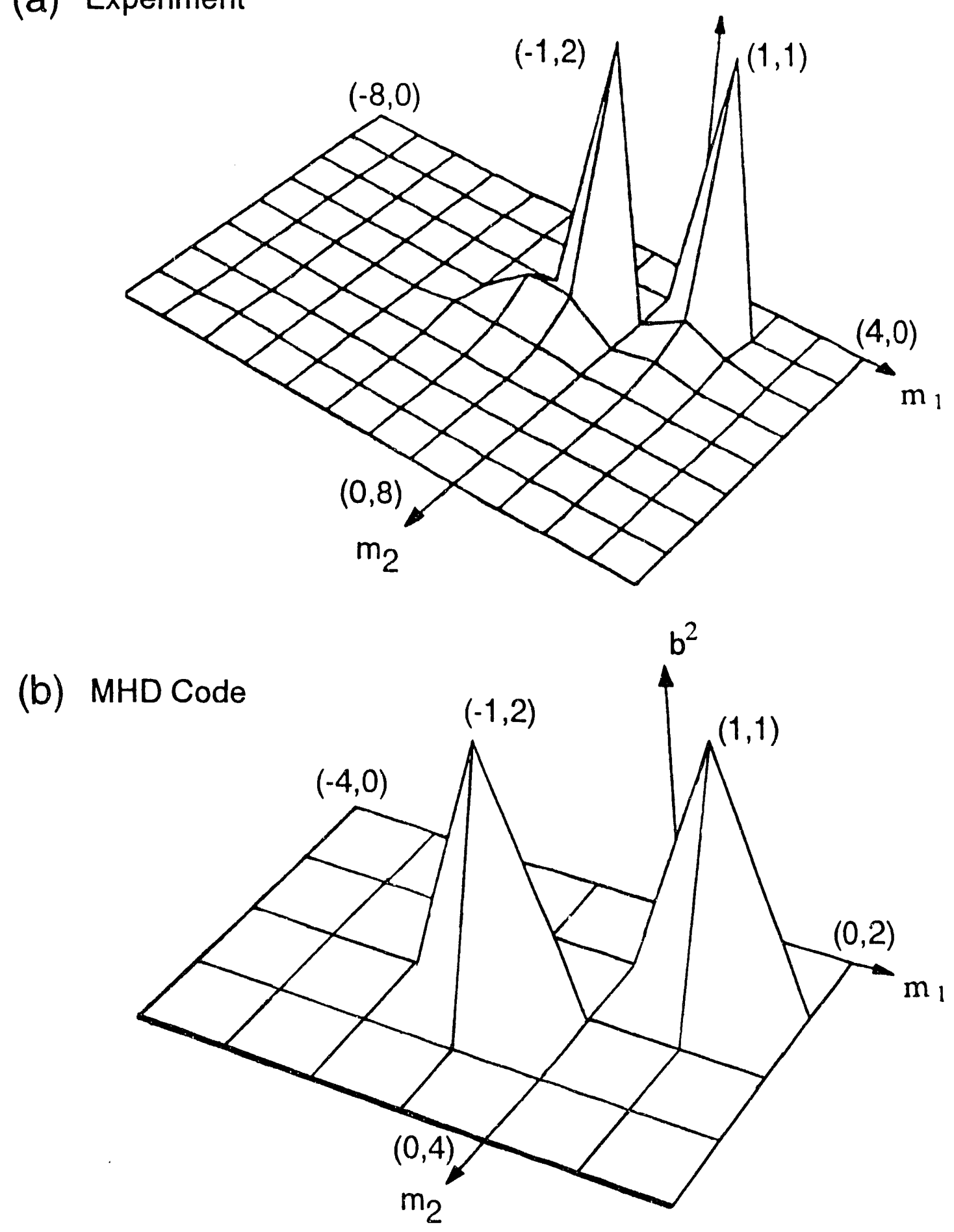

Fig. 3 
(a) Experiment

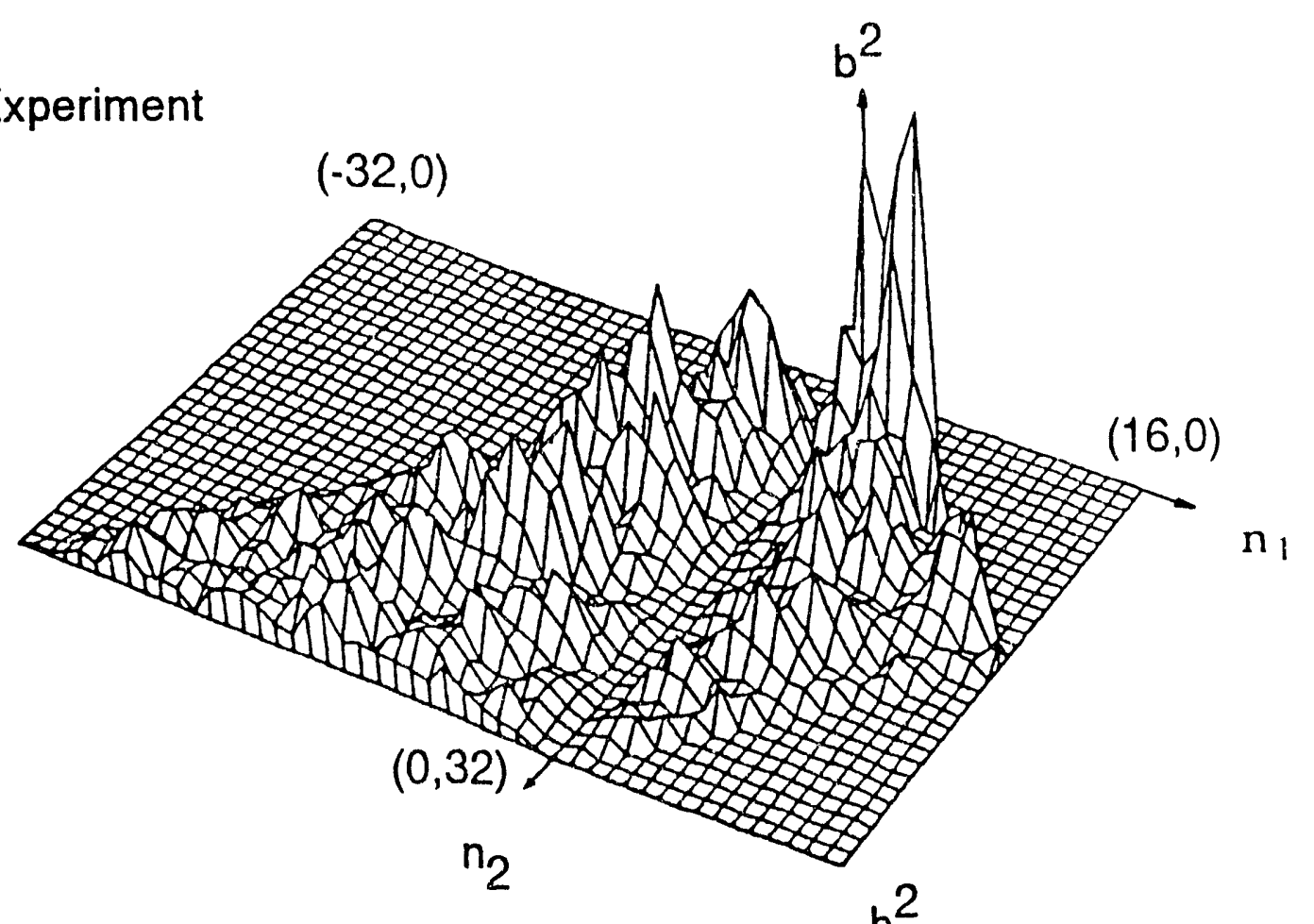

(b) MHD Code

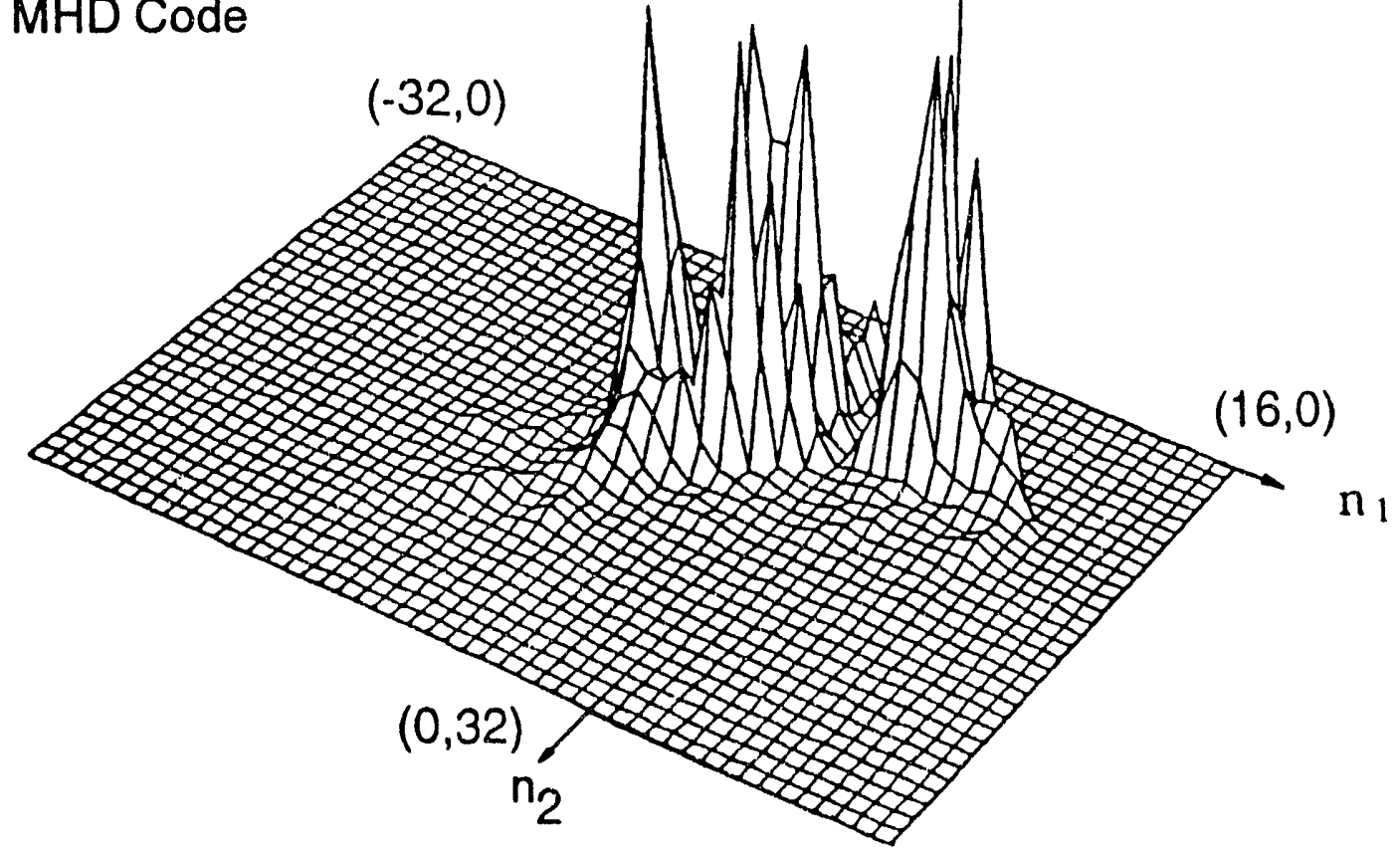

Fig. 4 
(a) Experiment

idal Mode Coupling

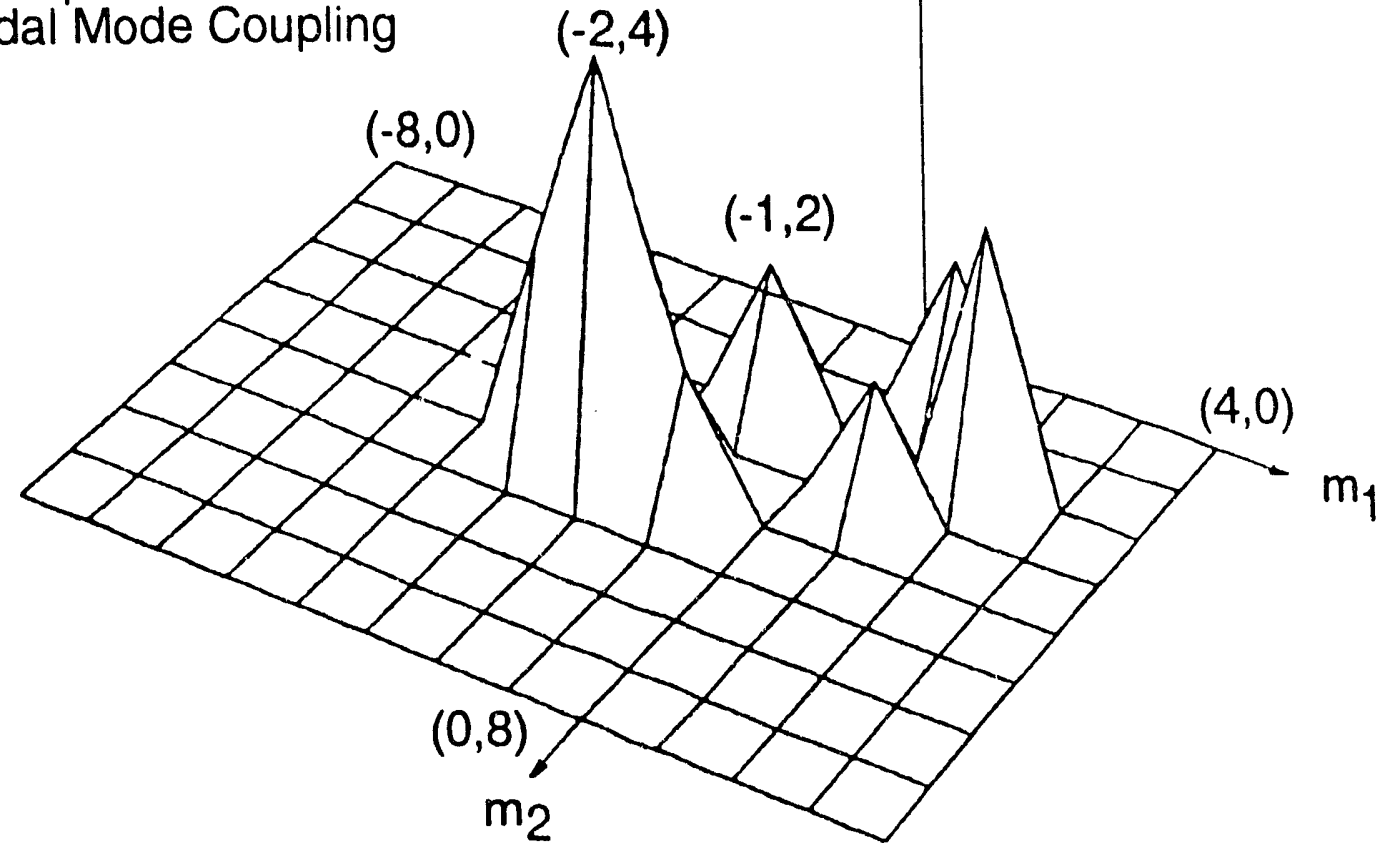

(b) Toroidal Mode Coupling

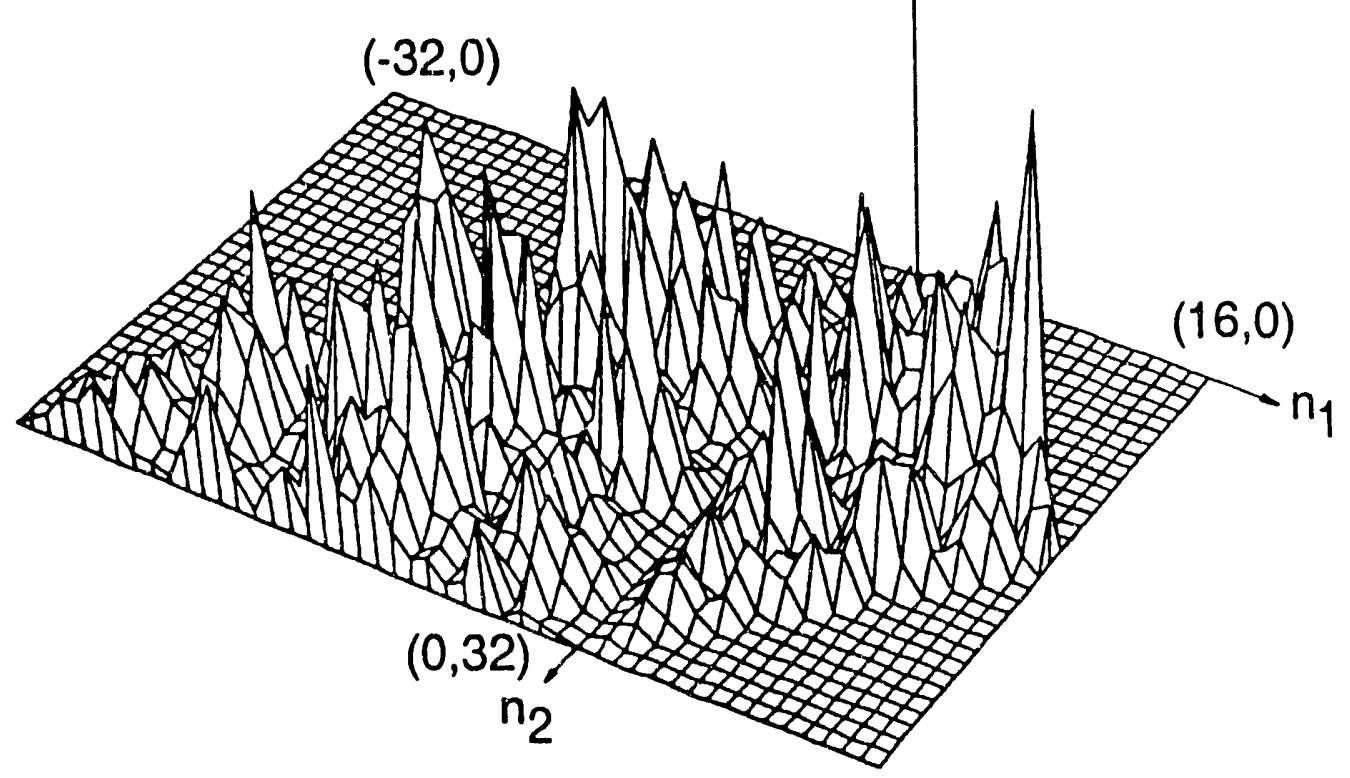

Fig. 5 


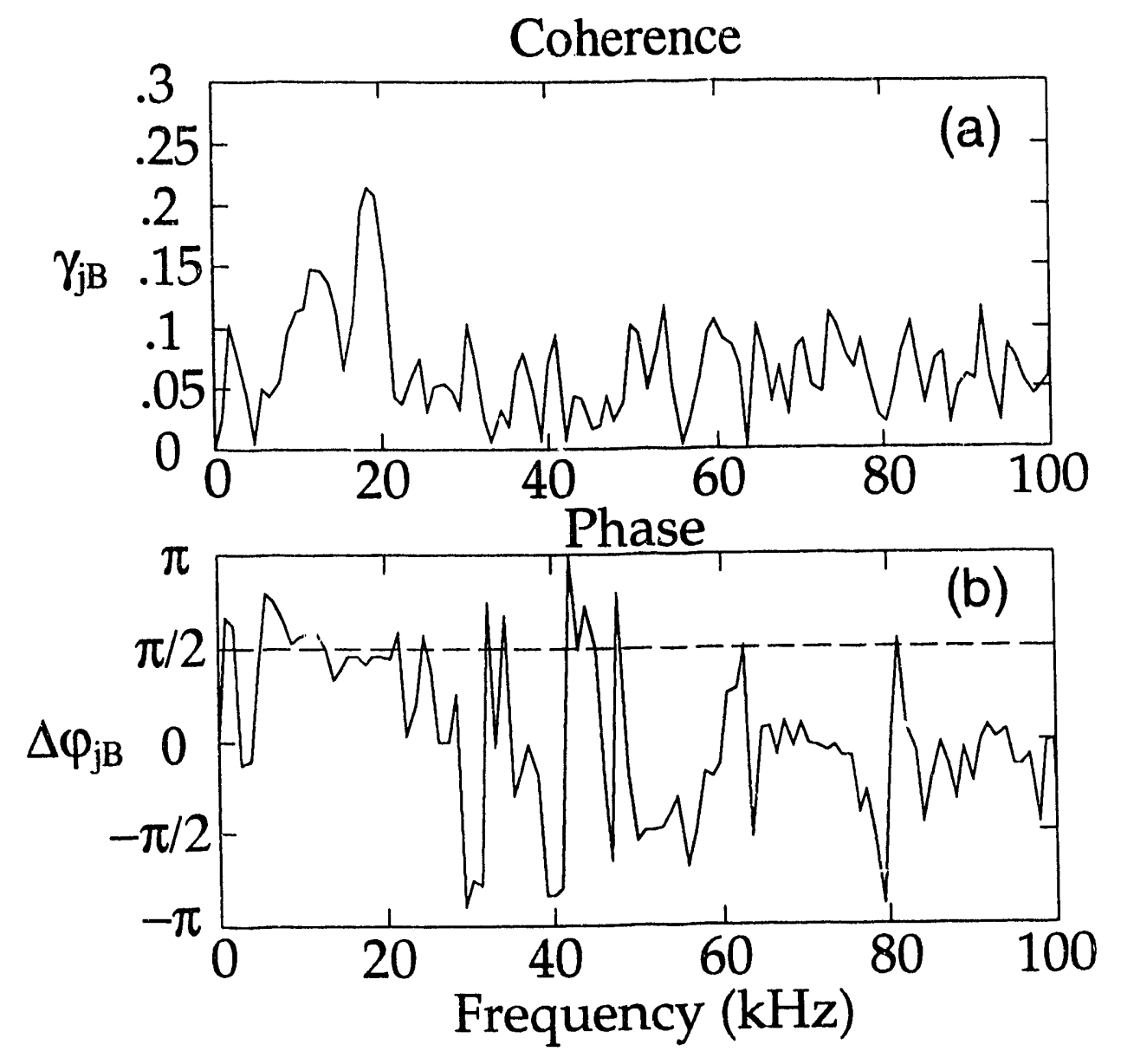

Fig. 6 


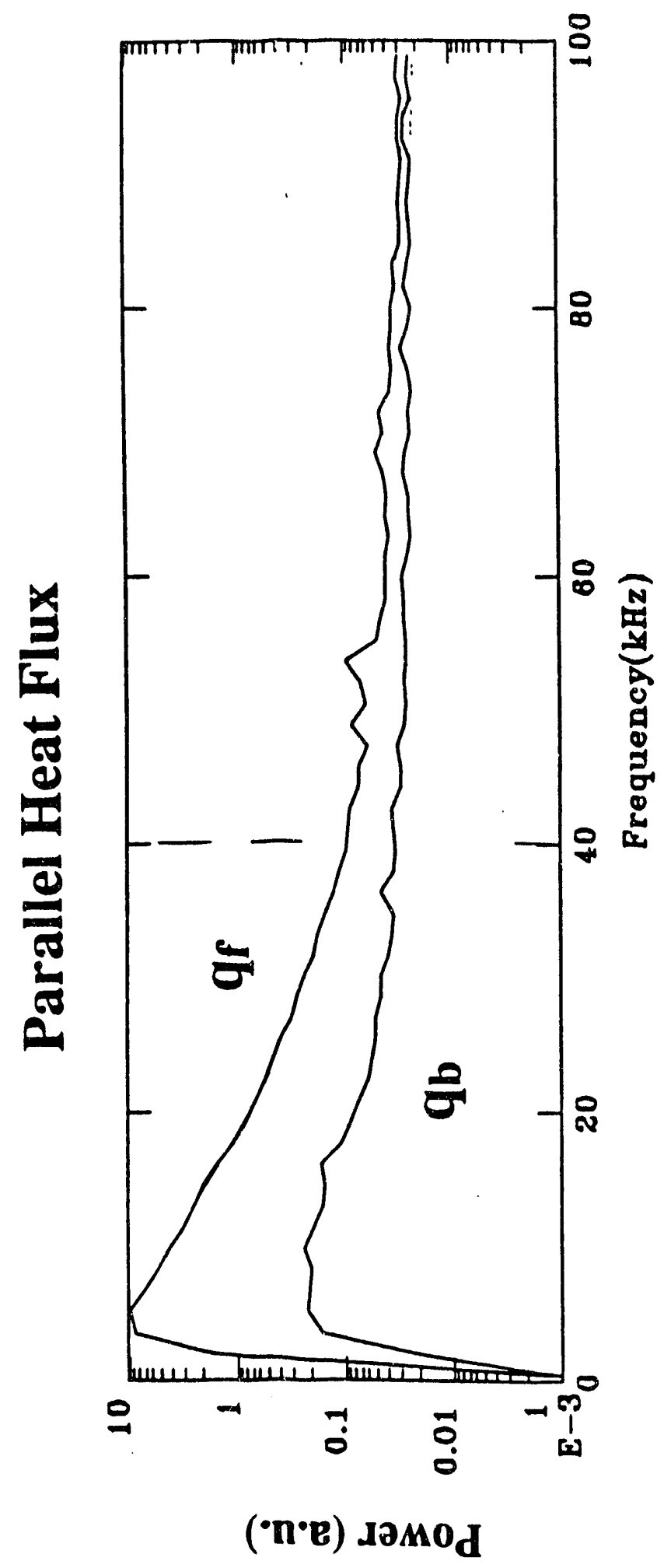

Fig. 7 

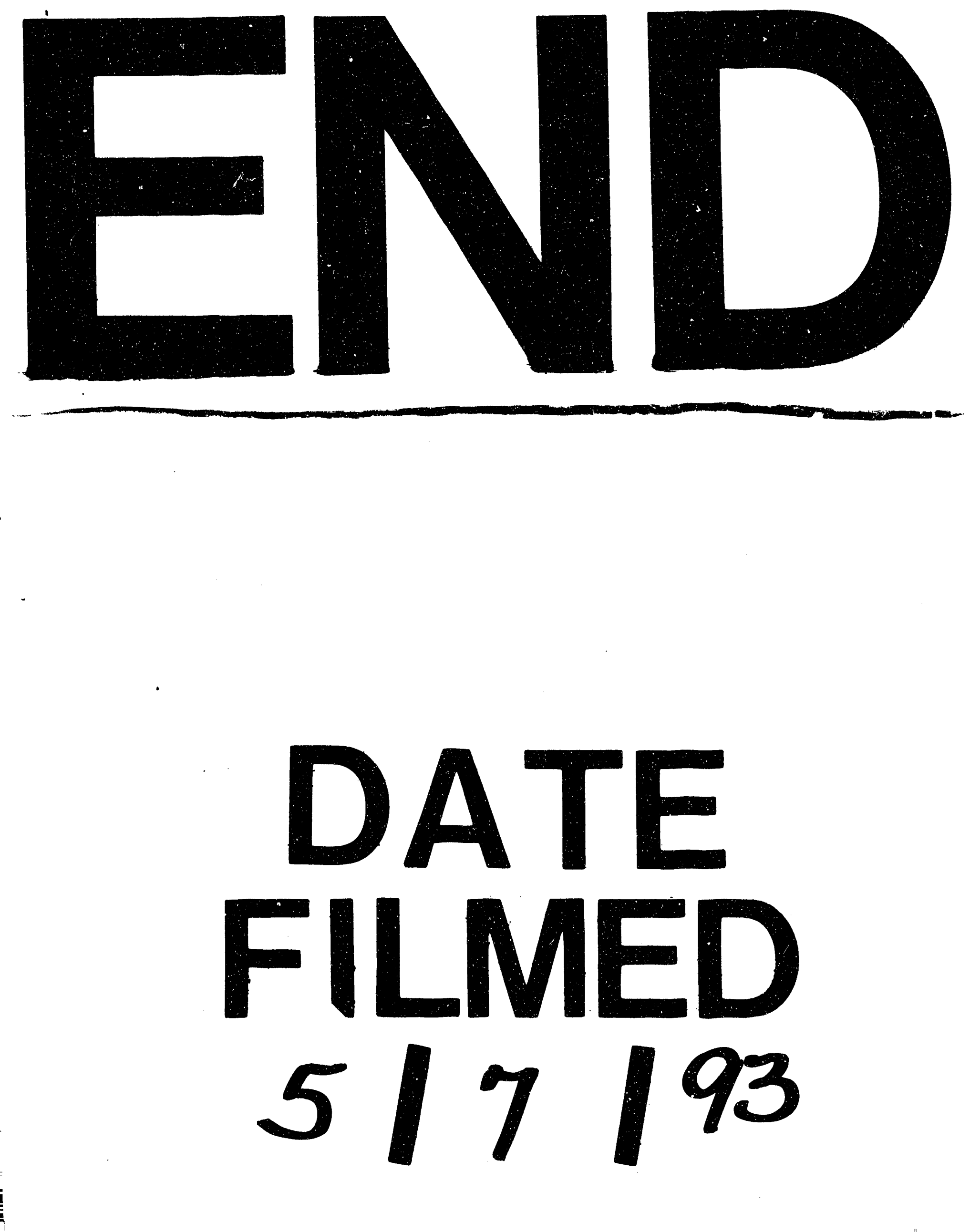